\title{
Trackability Filtering for Underactuated Systems
}

\author{
Eric E. Sandoz and Petar V. Kokotović and João P. Hespanha
}

\begin{abstract}
Underactuated systems are commonplace and present a challenge in designing tracking controllers. Foremost among these are vehicles, like passenger cars and aircraft. Such systems with more outputs than control inputs are not right invertible. As a result continuous control laws cannot, in general, be designed to track arbitrary reference signals. We present a Trackable Filter design which produces an augmented reference signal as close as possible to the original reference which the underactuated system can track with zero error. The resulting control law can be either discontinuous or continuous by the choice of the designer.
\end{abstract}

\section{INTRODUCTION}

As more effort is placed on control of autonomous vehicles there is a growing need for control design methods which are computationally efficient, robust and command feasible controls by actuators. Often, for cost reasons or simple historical inertia, many systems, vehicles in particular, are underactuated. Their physical actuators can only produce bounded continuous motion, and the entire position state of the vehicle is of interest. The last issue precludes the relegation of some states to zero dynamics [5], as their trajectories cannot be ignored. Model Predictive Control (MPC) can address all of these issues [3], [6], but at the cost of significant computational complexity. Switching methods [11] often require control actions which are not feasible by many actuators. One could solve inverse dynamics problems to find all possible signals that the system can track, but this is an extensive offline calculation. A simpler approach is to develop a filter which takes as input, a nominal tracking signal and produces an augmented version which the underactuated system can track with zero error. This paper proposes such a Trackable Filter design.

We will begin by looking at how we would design a tracking controller if our system was fully actuated. This will then guide us in dealing with the limitations imposed by an underactuated system.

For the following nonlinear system with output $x_{1}=h(\xi)$ which has uniform vector relative degree

$$
\begin{aligned}
\dot{\xi} & =f\left(\xi, u_{p}\right) \\
x_{1} & =h(\xi)
\end{aligned}
$$

This material is based upon work supported by the National Science Foundation under Grants CCR-0311084 and CNS-0720842.

J.P. Hespanha is with Faculty of Electrical and Computer Engineering, University of California, Santa Barbara, USA hespanha@ece.ucsb.edu

P.V. Kokotović is with Faculty of Electrical and Computer Engineering, University of California, Santa Barbara, USA petar ece.ucsb.edu

E.E. Sandoz is a graduate student at University of California, Santa Barbara, USA, esandoz@ece.ucsb.edu with state $\xi$ and input $u_{p}$, who's normal form is

$$
\begin{aligned}
\dot{z} & =\phi(z, x) \\
\dot{x}_{1} & =x_{2} \\
\dot{x}_{2} & =x_{3} \\
& \vdots \\
\dot{x}_{r} & =L_{f}^{r} h\left(T^{-1}(x)\right)+L_{g} L_{f} h\left(T^{-1}(x)\right) u_{p}
\end{aligned}
$$

where $h(\xi) \in \mathbb{R}^{n}$, each $x_{i} \in \mathbb{R}^{n}, i=1, \ldots, r$ and $u_{p} \in$ $\mathbb{R}^{p}, p<n$. The system has uniform vector relative degree $r$. It is assumed that $L_{g} L_{f} h(\cdot)$ has uniform full column rank $p$ and is Lipschitz continuous on a domain $\mathbf{D}_{\mathbf{x}}$. Because $L_{g} L_{f} h(\cdot)$ is not square, it has no inverse and the system is not right invertible, thus tracking of arbitrary signals $Y_{1}(t) \in$ $\mathcal{C}^{r}$ is not possible.

The normal form of system (1), shown in (2), is produced through the diffeomorphism $x=T(\xi)$. Which is composed of the output function and the first $r-1$ multivariable Lie derivatives, where

$$
T(\xi)=\left[\begin{array}{c}
h(\xi) \\
L_{f} h(\xi) \\
\vdots \\
L_{f}^{r-1} h(\xi)
\end{array}\right]=\left[\begin{array}{c}
x_{1} \\
x_{2} \\
\vdots \\
x_{r}
\end{array}\right]
$$

See [7][6] for details. Only systems with input to state stable (ISS) zero dynamics will be addressed here, as the $z$ subsystem may be neglected henceforth without altering the control design.

When confronted with a system which is not right invertible as is the case when the dimension of $u_{p}$ is $p<n$ it is common to choose a new output function $\bar{h}\left(x_{1}\right) \in \mathbb{R}^{p}$. Then compute a new normal form for a system of dimension $r p$ which is right invertible, leaving an $(n-p) r$ dimensional system of zero dynamics. How does one choose which states or combination of states to ignore when picking $\bar{h}\left(x_{1}\right)$ and will the zero dynamics be stable? What if one cares about all states in $x_{1}$ and has performance goals for them? A good example of this problem exists in both ground and aerial vehicles. In ground vehicles the position states consist of $X$ and $Y$ position in a plane and a heading or yaw angle $\psi$. It is commonplace in the active steering/steering control literature [3] to consider only the lateral deviation state $Y$ and heading angle $\psi\left(\bar{h}=\left[\begin{array}{ll}Y & \psi\end{array}\right]^{T}\right)$ and to ignore $X$ (it is typically assumed that $X$ is controlled by the driver). For autonomous vehicles this is an unnecessary restriction. Similarly, for aerial vehicles it is commonplace to push the roll and pitch angle dynamics into the zero dynamics, as for most maneuvers these states should be bounded in the 
neighborhood of zero. But when a loop or a roll maneuver is commanded these states become the ones which must track a reference and others become less important. Our approach is to provide a tool which can smoothly handle all of these cases without moving to a discontinuous control design [11].

We wish to solve a tracking problem and assume that we have a reference signal $Y_{1}(t)$ and its first $r$ time derivatives. Ideally, our goal is to choose a control law for $u_{p}$ such that $x_{1}(t) \rightarrow Y_{1}(t)$ as $t \rightarrow \infty$ (knowing that this is not possible for general non-right invertible systems). Let us, for convenience, name the reference signal and its derivatives accordingly

$$
\begin{aligned}
\dot{Y}_{i} & :=Y_{i+1}, i=1, \ldots, r-1 \\
\dot{Y}_{r} & :=Y_{u}
\end{aligned}
$$

Which is in the same block chain of integrators form as (2). To solve the tracking problem we form the tracking error coordinates as

$$
e_{i}:=x_{i}-Y_{i}, i=1, \ldots, r
$$

Then our tracking error system becomes

$$
\begin{aligned}
\dot{e}_{1} & =e_{2} \\
\dot{e}_{2} & =e_{3} \\
& \vdots \\
\dot{e}_{r} & =F_{r}(x)+G_{r}(x) u_{p}
\end{aligned}
$$

where

$$
\begin{aligned}
x & :=\left(x_{1}^{T} x_{2}^{T} \cdots x_{r}^{T}\right)^{T} \\
e & :=\left(e_{1}^{T} e_{2}^{T} \cdots e_{r}^{T}\right)^{T} \\
F_{r}(x) & :=L_{f}^{r} h\left(T^{-1}(x)\right), G_{r}(x):=L_{g} L_{f} h\left(T^{-1}(x)\right)
\end{aligned}
$$

Henceforth, a vector without a numbered subscript like $x$ refers to the stack of all of the $n$-dimensional sub-state vectors as in (7).

Choose a feedback gain matrix $K_{e}=$ $\left[\begin{array}{llll}K_{e 1} & K_{e 2} & \cdots & K_{e r}\end{array}\right], \quad K_{e i} \in \mathbb{R}^{n \times n}$ such that the matrix

$$
A_{e}=\left[\begin{array}{ccccc}
0 & I_{n} & 0 & \cdots & 0 \\
0 & 0 & I_{n} & & \vdots \\
& & & \ddots & I_{n} \\
K_{e 1} & K_{e 2} & K_{e 3} & \cdots & K_{e r}
\end{array}\right]
$$

is Hurwitz and $P_{e}=P_{e}^{T}>0$ is the solution to the Lyapunov equation $P_{e} A_{e}+A_{e}^{T} P_{e}=-Q_{e}$ for some positive definite symmetric matrix $Q_{e}$.

At this point if our system was fully actuated then our control signal would be $\hat{u}_{p} \in \mathbb{R}^{n}$ (as opposed to our actual control $\left.u_{p} \in \mathbb{R}^{p}, p<n\right)$. Then $G_{r}(x)$ which is full column rank by assumption is thus uniformly invertible over all $x$ in a domain of interest. We would then follow the feedback linearization control approach [7] choosing our control law to be

$$
\hat{u}_{p}=G_{r}(x)^{-1}\left(K e-F_{r}(x)\right)
$$

Which would produce the following exponentially stable error system

$$
\dot{e}=A_{e} e
$$

Which, for the positive definite radially unbounded Lyapunov function $V_{e}=e^{T} P_{e} e$ would produce the following Lyapunov function derivative

$$
\dot{V}_{e}=2 e^{T} P_{e} A_{e} e=-e^{T} Q_{e} e<0, \forall e \neq 0
$$

Which implies that $V(t)$ converges to zero. Since $Q_{e}$ is positive definite, our tracking error $e_{1}(t) \rightarrow 0$ as $t \rightarrow \infty$. The fully actuated (right invertible) case was examined to guide our later design of the trackabe filter.

Unfortunately our actual control signal is not of dimension $n$, the matrix $G_{r}(x)$ is not square and thus its inverse does not exist. We cannot implement the control law of (9) as a result. However, we will use (9) with our modified Trackable Filter system presented in the next section.

This paper is organized into six sections: Section 2 discusses the concept of Trackability Filtering followed by Section 3 which develops the control law for the Trackable Filtering concept and stability conditions. Section 4 presents a simulation example of the design method applied to a standard front wheel steering car model. Future work is presented in Section 5 and concluding remarks are made in Section 6.

\section{TrackABility Filtering CONCEPT}

Systems with $n$ outputs and $p<n$ inputs are not right invertible and continuous control laws cannot be designed to track any arbitrary reference signal $Y_{1}(t) \in \mathcal{C}^{r}$. The best that can be hoped for, with a continuous control law, is to follow $Y_{1}(t)$ as closely as possible and maintain a bounded tracking error. Furthermore, we would like to design a controller which allows the control designer the freedom to choose in which states or combination of states the tracking error is injected. In [1] a method utilizing nonlinear damping for dealing with this problem is presented but does not allow as much freedom to control to which states the tracking error goes.

The trajectory of $x_{1}(t)$ will lie on a $p$-dimensional manifold in $n$-dimensional space. Ignoring the ISS zero dynamics of (2) we construct a new normal form with the output function $\bar{h}\left(x_{1}\right) \in \mathbb{R}^{p}$. We use the same form of the coordinate transformation $T(x)$ as in (3) and the control law (9), with the only difference being dimension. The result is an exponentially stable tracking error system and zero dynamics $\bar{\phi}(\bar{z}, e, Y)$ which possess unknown stability properties and are driven by the reference signal $Y$ and error state $e$ which act as a disturbances to the zero dynamics.

$$
\begin{aligned}
\dot{\bar{z}} & =\bar{\phi}(\bar{z}, e, Y) \\
\dot{\bar{e}} & =\bar{A}_{e} \bar{e}
\end{aligned}
$$

Only the error vector output $e$ can be controlled. After the initial transient, $e=0$ and $\dot{\bar{z}}=\bar{\phi}(\bar{z}, 0, Y)$. From this it is clear that the zero dynamics are completely determined by 
signals outside our control. Since this is true of any choice of $\bar{h}\left(x_{1}\right)$ it is clear that we can only control our system output in an $p$-dimensional manifold. What we would like to do is construct a reference signal lying within this manifold which is as close as possible to our nominal reference signal. Such a signal can be tracked with zero error and represents the best that our underactuated system can do.

Let $Y_{1}(t)$ be the nominal reference signal which we would like to track, but which may not lie within our trackable $p$ dimensional manifold. Then let the trackable reference signal be described as

$$
Y_{T, 1}=Y_{1}+d_{1}
$$

where $d_{1}$ represents the vector between $Y_{1}$ and the trackable manifold, and is the output of an ISS relative degree $r$ filter.

$$
\begin{aligned}
\dot{d}_{1} & =d_{2} \\
\dot{d}_{2} & =d_{3} \\
& \vdots \\
\dot{d}_{r} & =K_{d} d+u_{d}
\end{aligned}
$$

where $u_{d} \in \mathbb{R}^{n}$ is a control term to be chosen later. The Trackable Filter concept simply produces a filter which generates, from the nominal reference $Y_{1}(t)$ a signal $Y_{T, 1}(t)$ which lies within the trackable $p$-dimensional manifold of motion for our plant, and the plant controller $u_{p}$ is designed to track $Y_{T, 1}(t)$.

\section{Constructive Trackable Filter Design}

The design begins by constructing new error variables. Let

$$
\begin{aligned}
Y_{T, i} & =Y_{i}+d_{i} \\
e_{i} & =x_{i}-Y_{T, i}=x_{i}-Y_{i}-d_{i}
\end{aligned}
$$

Then our error system becomes

$$
\begin{aligned}
\dot{e}_{1} & =e_{2} \\
\dot{e}_{2} & =e_{3} \\
& \vdots \\
\dot{e}_{r} & =F_{r}(x)+G_{r}(x) u_{p}-Y_{u}-K_{d} d-u_{d}
\end{aligned}
$$

From the stabilizing control law (9), we must render the right hand side of (16) equal to $K_{e} e$.

$$
F_{r}(x)+G_{r}(x) u_{p}-Y_{u}-K_{d} d-u_{d}=K_{e} e
$$

as that would make (16) equivalent to the exponentially stable system (10) which guarantees that $x_{1}(t) \rightarrow Y_{T, 1}(t)$ as $t \rightarrow \infty$. Since $u_{p} \in \mathbb{R}^{p}, p<n$ lacks sufficient degrees of freedom on its own to accomplish this task, we must use the additional control term $u_{d}$. Fortunately, $u_{d} \in \mathbb{R}^{n}$ thus we have an overdetermined problem and can satisfy condition (17) in multiple ways.

The term $u_{d}$ must be chosen carefully as it will act as a disturbance to the ISS trackability filter. Since $u_{d}$ will be a function of $d$, an improper choice can render the trackability filter unstable.
Intuitively, the plant control signal $u_{p}$ should do as much work as possible, thus minimizing the disturbance $u_{d}$. The disturbance to the trackability filter will drive $d_{1}$ away from zero, increasing the error between the reference that the plant can track versus the reference it is desired to track. For clarity let

$$
\begin{aligned}
G_{r}(x) u_{p}-u_{d} & =-F_{r}(x)+Y_{u}(t)+K_{d} d+K_{e} e \\
& =v(e, d, t)
\end{aligned}
$$

The plant control $u_{p}$ can only address the components of $v$ which lie in the range space of $G_{r}(x)$. Let $G_{r}^{\perp}$ be orthogonal to $G_{r}$ and each column of $G_{r}^{\perp}$ be of unit length. The set of vectors $\left[G_{r} G_{r}^{\perp}\right]$ forms a basis set, enabling us to perform a decomposition of $v$. Rewrite $v$ as

$$
\begin{aligned}
v & =G_{r} v_{p}+G_{r}^{\perp} v_{d} \\
G_{r}^{\perp^{T}} G_{r} & =0_{n-p \times p} \\
G_{r}^{\perp^{T}} G_{r}^{\perp} & =I_{n-p}
\end{aligned}
$$

We now have

$$
\begin{aligned}
G_{r} u_{p}-u_{d} & =G_{r} v_{p}+G_{r}^{\perp} v_{d} \\
v_{d} & =G_{r}^{\perp} v \\
v_{p} & =\left(G_{r}^{T} G_{r}\right)^{-1} G_{r}^{T} v
\end{aligned}
$$

The term $u_{d}$ must account for $G_{r}^{\perp} v_{d}$, since $u_{p}$ cannot. We wish to describe $u_{d}$ in a general way which will ensure this property, while clearly indicating any remaining design freedom. Let $u_{d}=H v$ where $H \in \mathbb{R}^{n \times n}$. One way to choose $H$ is as a minimal rank matrix, which ensures that disturbance energy gets injected in only $n-p$ directions.

$$
H=\left(G_{r} b-G_{r}^{\perp}\right) G_{r}^{\perp^{T}}
$$

Which, after substituting (23) into $u_{d}=H v$, yields

$$
\begin{aligned}
u_{d} & =H v \\
& =\left(G_{r} b-G_{r}^{\perp}\right) G_{r}^{\perp^{T}}\left(G_{r} v_{p}+G_{r}^{\perp} v_{d}\right) \\
& =\left(G_{r} b-G_{r}^{\perp}\right) v_{d}
\end{aligned}
$$

Which clearly shows that this choice of $H$ accounts for any component of $v$ which is orthogonal to $G_{r}$. Furthermore, the matrix $b \in \mathbb{R}^{p \times n-p}$ represents our remaining design freedom. Substitute (24) into (20) to solve for our two control terms

$$
\begin{aligned}
G_{r} u_{p}-\left(G_{r} b-G_{r}^{\perp}\right) v_{d} & =G_{r} v_{p}+G_{r}^{\perp} v_{d} \\
G_{r}\left(u_{p}-v_{p}-b v_{d}\right) & =0 \\
u_{p} & =v_{p}+b v_{d}
\end{aligned}
$$

In summary, the trackability filter design begins by computing the matrix $G_{r}^{\perp}$, then partitioning the vector $v$ according to the basis $\left[G_{r} G_{r}^{\perp}\right]$. Then choose the control law $u_{d}=H v$ and finally compute the plant control law from $G_{r} u_{p}=$ $(I+H) v$. Note that there are many ways to chose $H$. For 
our minimal rank choice given in (23), the design goes as follows

$$
\begin{aligned}
v & =-F_{r}(x)+Y_{u}(t)+K_{d} d+K_{e} e \\
& =G_{r} v_{p}+G_{r}^{\perp} v_{d} \\
v_{d} & =G_{r}^{\perp} v \\
v_{p} & =\left(G_{r}^{T} G_{r}\right)^{-1} G_{r}^{T} v \\
u_{d} & =\left(G_{r} b-G_{r}^{\perp}\right) v_{d} \\
u_{p} & =v_{p}+b v_{d}
\end{aligned}
$$

For some choice of matrix $b$. At this point we turn our attention to stability, which will help guide the choice of $b$.

\section{A. Stability Analysis}

Recall that the purpose of choosing $u_{p}$ and $u_{d}$ as in (30) and (31) was to render the tracking error system (16) exponentially stable. Our control choices satisfy (17), hence

$$
\begin{aligned}
\dot{e}_{1} & =e_{2} \\
\dot{e}_{2} & =e_{3} \\
& \vdots \\
\dot{e}_{r} & =K_{e} e
\end{aligned}
$$

which is exponentially stable, and equivalent to $\dot{e}=A_{e} e$, where $A_{e}$ is given in (8). The trackability filter system is now

$$
\begin{aligned}
\dot{d}_{1} & =d_{2} \\
\dot{d}_{2} & =d_{3} \\
& \vdots \\
\dot{d}_{r} & =K_{d} d+H v
\end{aligned}
$$

Which we can rewrite as

$$
\begin{aligned}
\dot{d} & =A_{d} d+B_{d} \Delta \\
B_{d} & =\left[\begin{array}{c}
0 \\
\vdots \\
I_{n}
\end{array}\right] \\
\Delta & =H\left(-F_{r}(e+Y+d)+Y_{u}+K_{d} d+K_{e} e\right)
\end{aligned}
$$

where $A_{d}$ is chosen exponentially stable via $K_{d}$, and $P_{d}=$ $P_{d}^{T}>0$ is the unique positive definite solution to the Lyapunov equation $P_{d} A_{d}+A_{d}^{T} P_{d}=-Q_{d}$ for some positive definite symmetric matrix $Q_{d}$.

Our last step is to ensure that the trackability filter is stable given our choice of $u_{d}$. The filter must remain bounded with respect to bounded input $Y_{u}(t) \in \mathcal{L}_{\infty} \cap \mathcal{C}^{0}$ and exponentially decaying input $e(t) \in \mathcal{L}_{2} \cap \mathcal{L}_{\infty}$. Of clear concern are the terms $H K_{d} d$ and $H F_{r}(e+Y+d)$ which are potentially working against the stabilizing term $K_{d} d$. Our choice of matrix $H$ has rank $n-p$, thus has $p$ eigenvalues at 0 . It is constructed as the outer product of two $n \times n-p$ matrices, hence its nonzero eigenvalues are

$$
G_{r}^{\perp^{T}}\left(G_{r} b-G_{r}^{\perp}\right)=-G_{r}^{\perp^{T}} G_{r}^{\perp}=-I_{n-p}
$$

It is helpful that $\|H\|=I$ which is independent of $b$.

We now turn our attention to the following Lyapunov function $V=e^{T} P_{e} e+d^{T} P_{d} d$ and its derivative along the trajectories of $e$ and $d$.

$$
\begin{aligned}
\dot{V}= & -e^{T} Q_{e} e-d^{T} Q_{d} d \\
& +2 d^{T} P_{d} B_{d} H\left(-F_{r}+Y_{u}+K_{d} d+K_{e} e\right)
\end{aligned}
$$

The product $P_{d r}=P_{d} B_{d}$ selects the last $n$ columns of $P_{d}$, or the $r^{\text {th }}$ block column of $P_{d}$. Note, that by appropriate initialization of $d(0)=x(0)-Y(0)$, then $e(0)=0$, hence for stability analysis, we can ignore all terms containing $e$. We will look for conditions on $b$ to make $\dot{V} \leq 0$ for $d$ bounded below by some constant. We will use the following definitions and bounds

$$
\begin{aligned}
\left\|F_{r}(x)\right\| & \leq L|x| \leq L(\bar{Y}+|d|) \\
|Y(t)| & \leq \bar{Y},\left|Y_{u}(t)\right| \leq \bar{Y}_{u}, \forall t \geq 0 \\
c_{1} & =2\left\|P_{d r}\right\| \\
c_{2} & =2\left\|P_{d r}\right\| L,(\|H\|=1) \\
2 d^{T} P_{d r} H K_{d} d & \leq c_{3}|d|^{2}
\end{aligned}
$$

by the assumption that $F_{r}(x)$ is Lipschitz, hence $\left\|F_{r}(x)\right\| \leq$ $L|x|$ on some domain $\mathbf{D}_{\mathbf{x}} \subset \mathbb{R}^{r n}$. Note that the Lipschitz rank bound is used here for generality, and can be overly conservative for some $F_{r}$. If a more accurate bounding function can be found, it should be used instead. The constant $c_{3}$ can be controlled to some degree by choice of $H$. Let $\mu_{e}, \mu_{d}>0$ be the smallest eigenvalues of $Q_{e}, Q_{d}$ respectively. Now we can upper bound our Lyapunov function derivative.

$$
\begin{aligned}
\dot{V} & \leq-\mu_{e}|e|^{2}-\left(\mu_{d}-c_{2}-c_{3}\right)|d|^{2}+c_{4}|d| \\
c_{4} & =c_{2} \bar{Y}+c_{1} \bar{Y}_{u}
\end{aligned}
$$

Stability requires that $c_{5}=\mu_{d}-c_{2}-c_{3} \geq 0$. Substituting and rearranging terms gives

$$
\dot{V} \leq-\mu_{e}|e|^{2}+\left(c_{4}-c_{5}|d|\right)|d|
$$

which, for $|d|>\frac{c_{4}}{c_{5}}$ we have $\dot{V}<0$. Thus stability is guaranteed if $c_{5} \stackrel{c_{5}}{>}$. At a minimum we must have $c_{2}<\mu_{d}, x \in \mathbf{D}_{\mathbf{x}}$, and $c_{3}<\mu_{d}-c_{2}$. While $c_{2}$ is system dependent, $\mu_{d}$ is a design choice however, $c_{3}$ can only be partially controlled by choice of $b$.

Lastly we must take a careful look at $H=\left(G_{r} b-\right.$ $\left.G_{r}^{\perp}\right) G_{r}^{\perp}{ }^{T}$ with respect to the term $d^{T} P_{d r} H K_{d} d$. Suppose that $d$ is such that $d^{T} P_{d r}=a^{T} G_{r}^{\perp^{T}}$, then we have

$$
\begin{aligned}
& d^{T} P_{d r} H K_{d} d=a^{T} G_{r}^{\perp^{T}} H K_{d} d \\
& =a^{T}{G_{r}^{\perp}}^{T}\left(G_{r} b-G_{r}^{\perp}\right) G_{r}^{\perp^{T}} K_{d} d \\
& =-a^{T} G_{r}^{\perp^{T}} K_{d} d
\end{aligned}
$$

Since $b$ has no effect in this situation, it is clear that for stability we require $-a^{T} G_{r}^{\perp^{T}} K_{d} d \leq c_{3}|d|^{2}$ and $c_{3}<\mu_{d}-$ $c_{2}, \forall d$ s.t. $d^{T} P_{d r}=a^{T} G_{r}^{\perp}$. For any $d$ not meeting the condition, we can choose a $b$ to add negativity. A stronger sufficient condition for stability is

$$
\begin{aligned}
-d^{T} P_{d r} G_{r}^{\perp} G_{r}^{\perp^{T}} K_{d} d & \leq c_{3}|d|^{2}, \forall x \in \mathbf{D}_{\mathbf{x}}, \forall d \\
c_{3} & <\mu_{d}-c_{2}
\end{aligned}
$$


This completes the conditions for a stable trackability filter design.

\section{B. Design Extensions}

One option, provided the $H$ matrix yields a stable system, is to choose $b$ statically such that disturbance energy is injected only into a subset of the $d$ states. This can be useful to the designer, in ensuring that a subset of states track the reference signal exactly.

One way to choose the matrix $b$ is to minimize the the complete disturbance term $\Delta=2 d^{T} P_{d r} H v$ directly. The same stability requirements (43) apply, let us expand $H$

$$
\begin{aligned}
\Delta & =a_{1}^{T}\left(G_{r} b-G_{r}^{\perp}\right) a_{2} \\
a_{1}^{T} & =2 d^{T} P_{d r} \\
a_{2} & =G_{r}^{\perp^{T}} v
\end{aligned}
$$

We wish to minimize $\Delta$, which intuitively will yield the best response. We can rewrite as

$$
\begin{aligned}
a_{1}^{T}\left(G_{r} b-G_{r}^{\perp}\right) a_{2} & =a_{3} \\
a_{1}^{T} G_{r} b a_{2} & =G_{r}^{\perp} a_{2}+a_{3}
\end{aligned}
$$

where $a_{3} \in \mathbf{R}$ is the value we would like the disturbance to be, ideally $a_{3} \leq 0$. However, since the system is underactuated it is not always possible to find a solve for $b$ from (49) for some $a_{3} \leq 0$. One can arbitrarily choose $a_{3}<0$ and use pseudoinverses to solve for $b$, which will minimize the disturbance when it can. This approach was found to yield a superior response in the car steering example presented next.

\section{EXAMPLE: Front WHEEL STEERING CAR}

An example of the Trackability Filtering design method presented above was applied to the car steering problem mentioned throughout the paper. We employ the Bicycle Model for steering dynamics [10].

Now examine the car steering problem as it fits the vectorial strict feedback form. (Rolling resistance is ignored).

$$
\begin{aligned}
& \dot{\xi}_{1}=\left[\begin{array}{ccc}
\cos \psi & -\sin \psi & 0 \\
\sin \psi & \cos \psi & 0 \\
0 & 0 & 1
\end{array}\right]\left[\begin{array}{c}
v_{u} \\
v_{v} \\
v_{\psi}
\end{array}\right] \\
& \dot{\xi}_{2}=\left[\begin{array}{c}
-C_{d} v_{u}^{2}+2 v_{v} v_{\psi} \\
-2 v_{u} v_{\psi}+c_{1} F_{v r}\left(\xi_{2}\right) \\
c_{3} F_{v r}\left(\xi_{2}\right)
\end{array}\right]+\left[\begin{array}{cc}
q_{1} & 0 \\
0 & q_{1} \\
0 & q_{2}
\end{array}\right]\left[\begin{array}{c}
F_{u} \\
F_{v f}
\end{array}\right] \\
& q_{1}=2 / m, q_{2}=2 l_{1} / I, q_{3}=-2 l_{2} / I
\end{aligned}
$$

where $\xi_{1}=(X Y \psi)^{T}$ and $m=1533 \mathrm{~kg}$ is the vehicle mass, $l_{1}=1.04 \mathrm{~m}$ is the distance from the center of gravity (CG) to the front axle, $l_{2}=1.65 \mathrm{~m}$ the distance from $\mathrm{CG}$ to rear axle, and $I=2712 \mathrm{~kg} \mathrm{~m}^{2}$ is the moment of inertia. A lumped parameter $C_{d}=0.000321$ contains the coefficient of aerodynamic drag and vehicle frontal cross-sectional area. Note that $G_{2}$ is a constant matrix.

An important point is our choice of the forward tire force $F_{u}$ and lateral tire force $F_{v f}$ as control variables. Of course wheel torque and wheel angle are the real control variables. We utilize the Pacejka tire model [13] to compute $F_{v r}$, which is a static nonlinear mapping between vehicle states, wheel angle and tire slip to tire forces $F_{u}$ and $F_{v f}$. For the region of normal operation, where the tire is not sliding, this function is invertible. Thus, provided our controller only commands forces achievable by the tire through a choice of wheel angle and torque (i.e. slip), then we are free to work with whichever variable is most convenient.

$$
\begin{aligned}
& \dot{\xi}_{1}=G_{1}\left(\xi_{1}\right) \xi_{2} \\
& \dot{\xi_{2}}=f_{2}\left(\xi_{2}\right)+G_{2} u_{p}
\end{aligned}
$$

The model is in strict feedback form which is easy to place in normal form through the diffeomorphism $x=T(\xi)$ based on the following Lie derivatives.

$$
\begin{aligned}
h\left(\xi_{1}\right) & =\xi_{1}=x_{1} \\
L_{f} h(\xi) & =G_{1} \xi_{1}=x_{2} \\
L_{f}^{2} h(\xi) & =\frac{\partial G_{1} \xi_{2}}{\partial \xi_{1}} G_{1} \xi_{2}+G_{1} f_{2} \\
L_{g} L_{f} h(\xi) & =G_{1} G_{2}
\end{aligned}
$$

Which gives us the normal form representation

$$
\begin{aligned}
& \dot{x}_{1}=x_{2} \\
& \dot{x}_{2}=F_{r}\left(T^{-1}(x)\right)+G_{r}\left(T^{-1}(x)\right) u_{p}
\end{aligned}
$$

We present two different approaches to choosing the control law. First, we choose the matrix (vector in this case) $b$ according to (49), such that the disturbance term is directly minimized. First, stability was verified for the weaker condition of (43) and since $G_{r}(\psi), \psi \in[-\pi, \pi]$ is a function of only one state variable, this was straight forward. The control laws were computed as given in (31). The simulation results are shown in Figs. 1 and 2. Fig. 1 shows the trajectory (solid

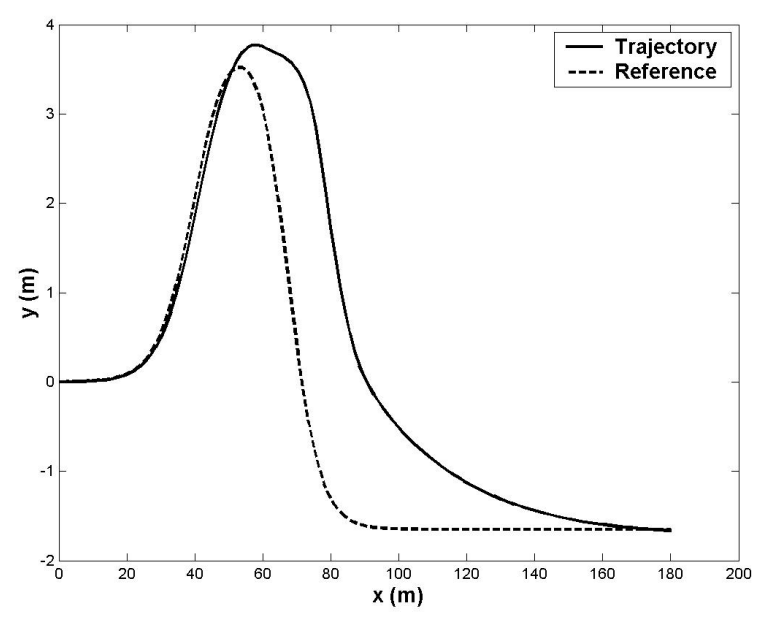

Fig. 1. Vehicle trajectory in the $(\mathrm{x}, \mathrm{y})$ plane. Heading is not shown.

line) of the vehicle compared to the reference (dashed line) for a double lane change maneuver. This maneuver is not trackable with zero error for the underactuated vehicle model. The maneuver definition is taken from [3]. Observe that the error trajectory of $X(t)$ goes negative, implying that the car slowed down in order make the hard right turn after the 

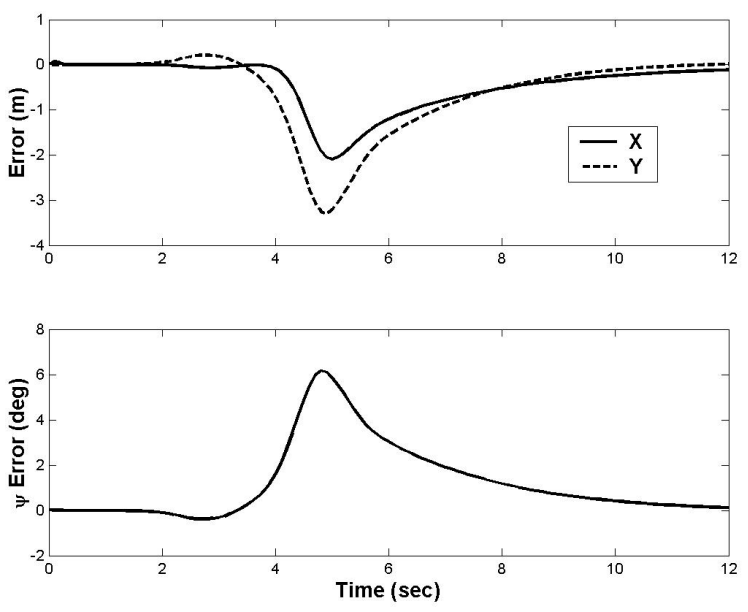

Fig. 2. Tracking error trajectory with respect to time. Top: Position error. Bottom: Heading angle error.

initial hard left turn. This makes intuitive sense for anyone with experience driving a car.

A different control law was implemented to show the versatility of the trackability approach. In this case $b$ was chosen such that the disturbance energy was injected only into the states associated with lateral position $Y(t)$. Fig. 3 shows the simulation results for this control law. Fig. 3
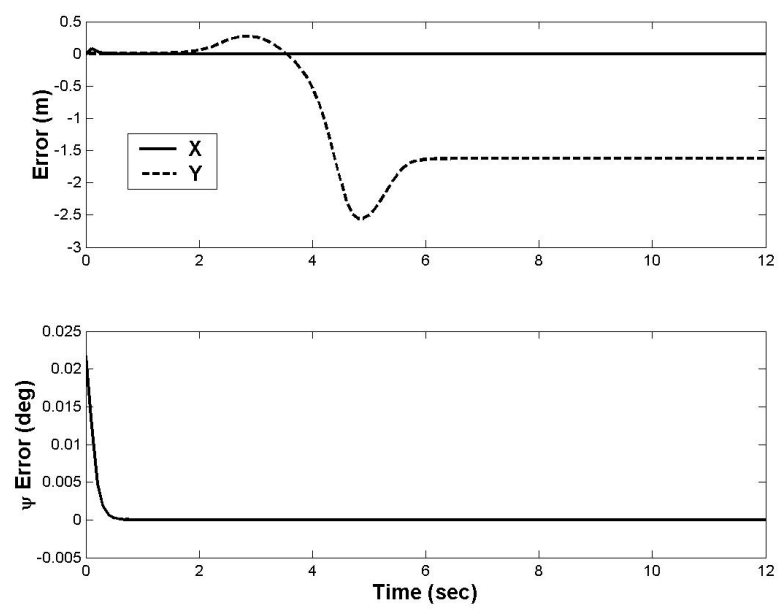

Fig. 3. Tracking error trajectory with respect to time with disturbance energy only injected into $Y(t)$. Top: Position error. Bottom: Heading angle error.

clearly shows that the angular error and $\mathrm{X}$ direction error exponentially converge to zero, while the only nonzero error is in the lateral deviation $Y(t)$.

\section{FUTURE WORK}

The next step is to extend the design to systems in strict feedback form (instead of normal form) with the nominal control law $\hat{u}_{p}$ chosen via backstepping instead of feedback linearization. Following that the trackability filtering design must be compared against MPC designs, as in [3] for the car steering example. It is of interest to know how our approach performs against the nearly optimal approach of finite horizon MPC. Implicit in this is to split the cost functional between our plant tracking error $e$ system and the reference trajectory error $d$ system. If the trackability filter approach performs well, as these simulation results suggest, it represents a far less computationally intensive approach to solving tracking problems for underactuated systems.

\section{Conclusions}

A Trackability Filter based tracking control design was developed and sufficient conditions for a stability were presented. The method was tested in simulation on a practical example of an underactuated system, a front wheel steering car. The method performed well and allowed the designer the freedom to choose where the tracking error got injected, demonstrating its practical utility.

\section{REFERENCES}

[1] E. E. Sandoz and P. V. Kokotović, Continuous Path Following Control for Underactuated Systems With Bounded Actuation, Proc. of Intelligent Vehicles Symposium 2007, June 13-15, Istanbul.

[2] R. Skjetne and T. I. Fossen and P. V. Kokotović, Robust Output Maneuvering for a Class of Nonlinear Systems, Automatica J., vol. 40, 2004, pp 272-283.

[3] P. Falcone and F. Borrelli and J. Asgari and H.E. Tseng and D. Hrovat, Predictive Active Steering Control for Autonomous Vehicle Systems, IEEE Transactions on Control Systems Technology, vol. 15, no. 3, May 2007, pp. 566-580.

[4] M. M. Seron and J. H. Braslavsky and P. V. Kokotović and and D. Q. Mayne, Feedback Limitations in Nonlinear Systems: From Bode Integrals to Cheap Control, IEEE Transactions on Automatic Control, vol. 44, no. 4, April 1999.

[5] M. M. Seron and J. H. Braslavsky and G. C. Goodwin, Fundamental Limitations in Filtering and Control, Springer, London; 1997.

[6] H. K. Khalil, Nonlinear Systems, Prentice Hall, New Jersey; 2002.

[7] A. Isidori, Nonlinear Control Systems, Springer, London; 1995.

[8] D. B. Dačić and M. V. Subbotin and P. V. Kokotović, Path-Following Approach to Control Effort Reduction of Tracking Feedback Laws, Automatica J., vol. 40, 2004, pp 373-383.

[9] D. B. Dačić and M. V. Subbotin and P. V. Kokotović, "Path-Following for a Class of Nonlinear Systems with Unstable Zero Dynamics",43rd IEEE Conf. on Decision and Control, 2004, pp 4915-4920.

[10] U. Kiencke and L. Nielsen, Automotive Control Systems, Springer, Berlin; 2000.

[11] F. Rehman, Discontinuous Steering Control for Nonholonomic Systems With Drift, Nonlinear Analysis, vol. 63, 2005, pp 311-325.

[12] M. Kristić and I. Kanellakopoulos and P. Kokotović, Nonlinear and Adaptive Control Design, Wiley Interscience, New York, 1995.

[13] E. Bakker and L. Nyborg and H. B. Pacejka, "Tyre Modelling for Use in Vehicle Dynamics Studies", SAE Technical Paper Series, 1987, pp $1-15$. 